\title{
Atmospheric Aerosols Detection Research with a Dual Field of View Lidar
}

\author{
Lv Lihui, ${ }^{1,2}$ Zhang Tianshu, ${ }^{1}$ Liu Cheng, ${ }^{2}$ Dong Yunsheng, ${ }^{1}$ Chen Zhenyi, \\ Fan Guangqiang, ${ }^{1}$ Liu Yang, ${ }^{1,2}$ and Liu Wenqing ${ }^{1,2}$ \\ ${ }^{1}$ Key Laboratory of Environmental Optics and Technology, Anhui Institute of Optics and Fine Mechanics, \\ Chinese Academy of Sciences, Hefei, Anhui 230031, China \\ ${ }^{2}$ University of Science and Technology of China, Hefei, Anhui 230031, China
}

Correspondence should be addressed to Liu Cheng; chliu81@ustc.edu.cn

Received 25 August 2015; Revised 5 December 2015; Accepted 16 December 2015

Academic Editor: Dean S. Venables

Copyright (C) 2015 Lv Lihui et al. This is an open access article distributed under the Creative Commons Attribution License, which permits unrestricted use, distribution, and reproduction in any medium, provided the original work is properly cited.

A dual field of view lidar system with two independent receivers is described to realize the detection of atmospheric aerosols. A CCD camera is attached to a backscatter lidar as a receiver to complement the data in the near-field range affected by the incomplete overlap between the laser beam and the receiver field of view. The signal detected by the CCD camera is corrected and finally glued with the signal of the backscatter lidar to retrieve the aerosol extinction coefficient with Fernald algorithm. The aerosol extinction profile and visibilities measured by the dual field of lidar had been compared with the results measured by another general backscatter lidar and a surface aerosol instrument, respectively. The results suggested that the dual field of view lidar based on a CCD camera is feasible and reliable. It could obtain the data both in the near and in the far range simultaneously, improving the detection accuracy of the lidar system effectively.

\section{Introduction}

The atmospheric aerosols, especially the aerosols nearground, have a large impact on the local and regional air quality and visibility, as well as the human health and public transport safety directly and indirectly. Therefore, it is of vital importance to detect the vertical distribution of atmospheric aerosols near the ground. As a powerful tool to detect the spatial distribution of atmospheric aerosols, lidar has been widely used in the field of atmospheric and environmental monitoring [1]. However, the incomplete overlap between the laser beam and the receiver field of view of the backscatter lidar significantly affects the observation of aerosol optical properties in the near-field range. So the backscatter lidar has a shortcoming in the lower hundreds of meters because of the geometric overlap factor [2]. In this paper, the problem is overcome by a dual field of view lidar based on a CCD camera.

Welsh and Gardner had verified the feasibility of a bistatic lidar that uses a CCD camera as the receiver in 1989 [3]. Then, the lidar based on a CCD camera is gradually used in the near-ground atmospheric aerosols detection [4-6]. However, the application of the standard charge-coupled device camera lidar is limited in the near range. In this paper, a CCD camera is attached to a backscatter lidar to expand the detection range of the atmospheric aerosols in the near range. The CCD camera with large FOV is mainly used to detect the atmosphere in the near range and the receiver of the backscatter lidar is mainly used to detect the atmosphere in the far range. At last, the signals of the two receivers are glued and used to retrieve the whole range aerosol extinction properties.

\section{System Structure}

The configuration of the dual field of view lidar is shown in Figure 1 and the key specifications are given in Table 1. As illustrated in Figure 1, the CCD camera and telescope are separated by a distance $D$ and the CCD camera is parallel to the axis of the telescope. The laser source was a pulsed Nd:YAG laser operating at a wavelength of $532 \mathrm{~nm}$ with a pulse repetition rate of $20 \mathrm{~Hz}$. The CCD camera with $3358 \times 2536$ active pixels was cooled to $-10^{\circ} \mathrm{C}$ to improve 
TABle 1: Key specifications of the dual field of view lidar.

\begin{tabular}{lcc}
\hline & Feature & Specification \\
\hline & Laser: Nd:YAG & \\
& Wavelength & $532 \mathrm{~nm}$ \\
Transmitter & Pulse energy & $200 \mathrm{~mJ}$ \\
& Repetition frequency & $20 \mathrm{~Hz}$ \\
Divergence & $0.5 \mathrm{mrad}$ \\
\hline CCD camera & \\
CCD:QHY9 & $3358 \times 2536$ \\
& Pixels & $(860 \mathrm{million})$ \\
& Pixel size & $5.4 \mu \mathrm{m} \times 5.4 \mu \mathrm{m}$ \\
Active area & $19.7 * 15.04 \mathrm{~mm}$ \\
Receiver & Peak Quantum Efficiency & $56 \%$ \\
& Wide-angle lens & \\
Angular field & $114^{\circ}$ \\
Focal length & $14 \mathrm{~mm}$ \\
Max aperture & $\mathrm{F} 2.8$ \\
Telescope: Cassegrain & $230 \mathrm{~mm}$ \\
Diameter & PMT \\
Detector & Photo counter \\
Data acquisition & $7.5 \mathrm{~m}$ \\
Range resolution &
\end{tabular}

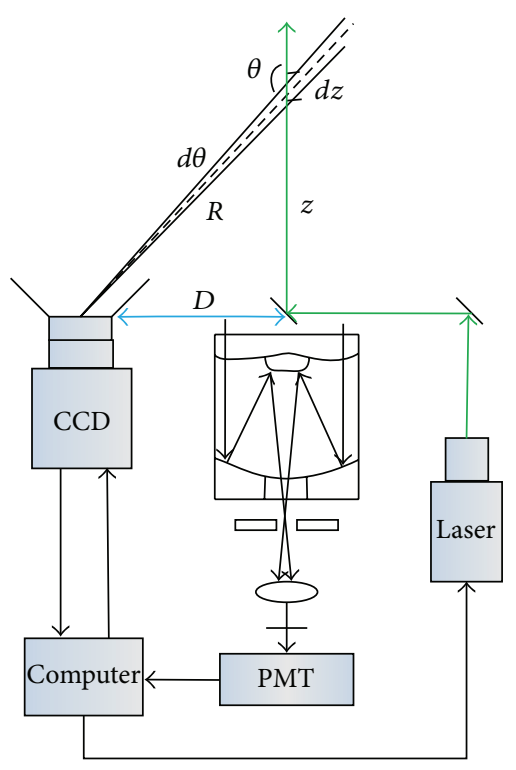

FIGURE 1: Configuration of the dual field of view lidar.

the resolution and reduce the noise. The FOV of the receiver which consists of a CCD and a wide-angle lens is about $75.4^{\circ}$.

In the dual field of view lidar system, the laser beam is emitted into the atmosphere vertically and then the scattered signal is detected by two receivers. The CCD camera records

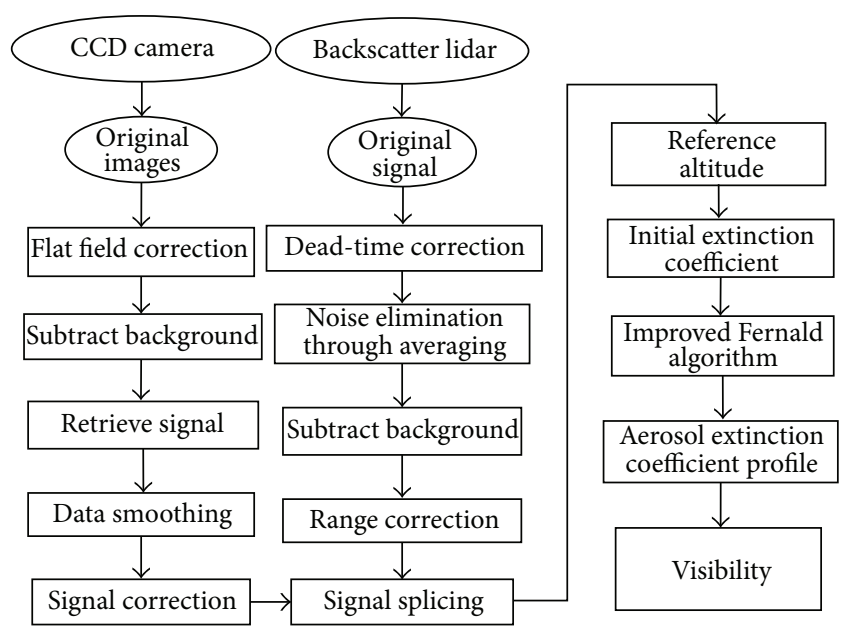

FIGURE 2: The flow chart of the data processing.

the signal in the form of image. From geometry, the resolution of the signal detected by the CCD camera is given simply by

$$
\begin{gathered}
z=-\frac{D}{\tan \theta}, \\
d z=\frac{R^{2} d \theta}{D},
\end{gathered}
$$

where $d \theta$ is the FOV of each pixel. The CCD camera with large FOV is mainly used to detect the signal in the near range while the backscatter lidar with small FOV is mainly used to detect the signal in the far range. Finally, the signals are glued to retrieve the aerosol profiles.

\section{Method}

In the dual field of view lidar technique, some important parameters should be assumed in the CCD camera data retrieval and the most significant one is the exposure time, which has an important influence on the signal-to-noise ratio (SNR). Wang et al. [7] have pointed out the relationship between the signal-to-noise ratio (SNR) and exposure time of an astronomical CCD camera. It revealed that the SNR of the astronomical cooled CCD was proportional to the exposure time $t$ when the exposure time $t<t_{c}$ ( $t_{c}$ is approximately $3 \mathrm{~s}$ ) while proportional to the square root of the exposure time $t$ when the exposure time $t>t_{c}$. Besides, according to the signal theory, the SNR is proportional to the square root of the number of repetitions. All the analyses show that signal exposure mode with longer exposure time is more suitable when the total exposure time is ascertained. However, the signal will be saturated if the exposure time is too long. The exposure time in our experiment is set as $50 \mathrm{~s}$.

The flow chart of the data processing is shown in Figure 2. First, a flat field calibration is applied to the images recorded by the CCD camera. Then the background is subtracted from the image with scattered signal to remove the influence of 


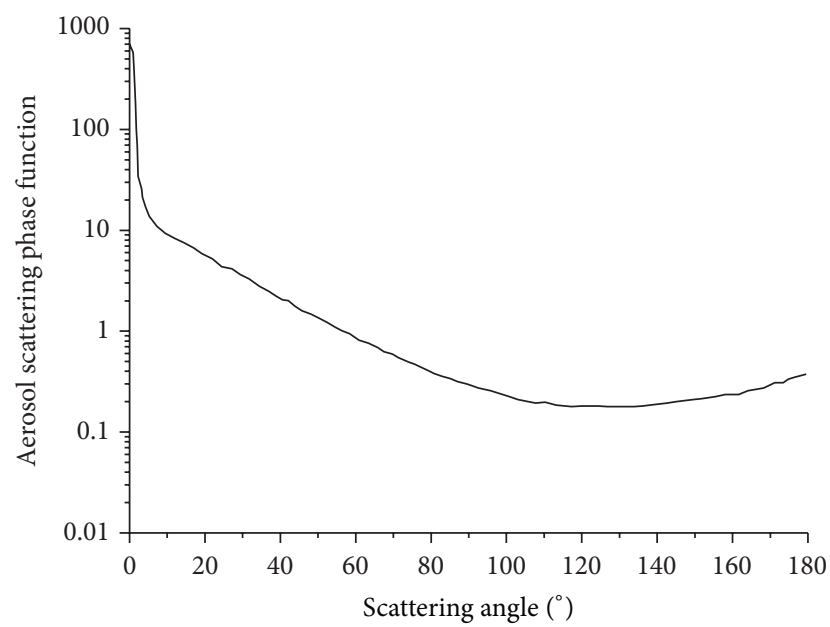

FIGURE 3: The curve of continental aerosol scattering phase function.

the background. The gray scale at each pixel is summed in the direction perpendicular to the telescope axis within a certain range to provide information of the atmospheric scattering. The altitude of the scattered signal is derived from the geometry of the laser and CCD camera locations. The signals detected by the CCD camera, unlike ones from the backscatter lidar, vary dramatically with the scattering angle $\theta$ (it is always $180^{\circ}$ for a backscatter lidar, whereas in the CCD technique, it varies with altitude), so the signals should be corrected based on the aerosol scattering phase function. Here we use the aerosol scattering phase function [8] (calculated from the second simulation of the satellite signal in the solar spectrum) which is shown in Figure 3. In the dual field of view lidar system, the CCD camera receives the scattering signal in the range $143^{\circ} \sim 180^{\circ}$. The signal detected by the CCD camera is converted to the $180^{\circ}$ with the aerosol scattering phase function and then glued with the signal acquired by the backscatter lidar.

The signal gluing theory is an ideal method to solve the problems of signal saturation in the near range and low SNR in the far range [9] in the backscatter lidar. Here the signal detected by the CCD camera and the photo counting signal detected by the backscatter lidar are glued. The main steps of the signal splicing procedure can be summarized as follows [10]: first, the fitting area where both the signals have higher SNR and better linearity should be selected; then the best gluing area is chosen with standards of justification to fit the two groups of signals and finally the signals are spliced with the parameters obtained from the linear fitting curve.

In order to guarantee the SNR of the signal in fitting area, the range of signals depends on the effective detection range of the CCD camera and the backscatter lidar. Some research has suggested that the distribution function of the laser beam images recorded by the CCD is characteristic of Gaussian curve, so the SNR at each altitude can be calculated with the Gaussian distribution formula [11]. The two-dimensional image of the laser beam is shown in Figure 4(a), in which the beam direction is parallel to the $y$ axis and the color level

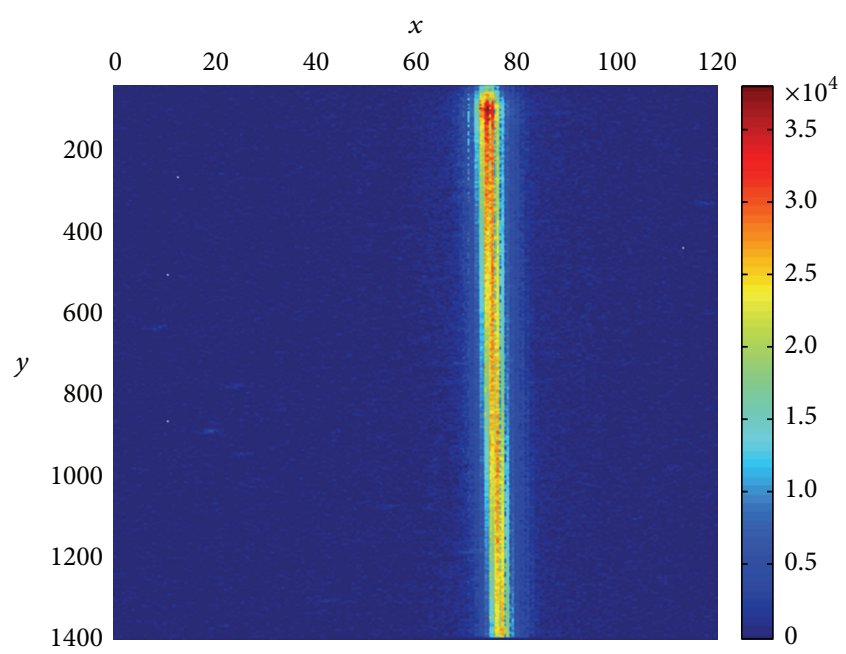

(a)

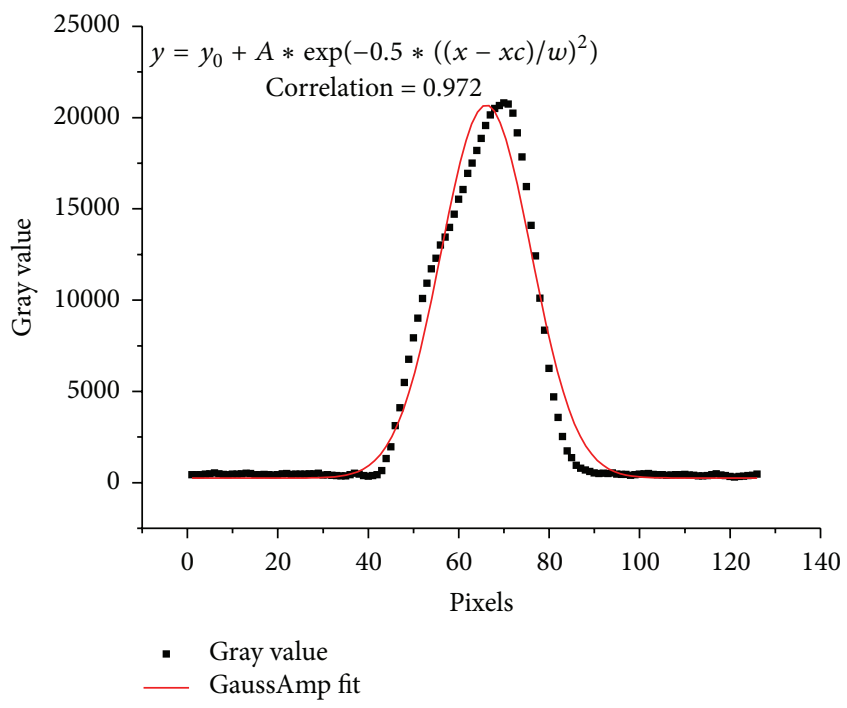

(b)

FIgure 4: (a) Two-dimensional image of the laser beam. (b) The scatter diagram of the pixels and its corresponding gray scales perpendicular to the direction of the laser beam.

represents the intensity of the received signal. Figure 4(b) shows the scatter diagram of the pixels and its corresponding gray scales at some attitude. We can obtain the SNR of the signal received by the CCD camera by fitting the scatter plot with Gaussian formula. The SNR can be calculated as

$$
\mathrm{SNR}=\frac{N_{s}}{N_{n}}=\frac{\sqrt{2 \pi} A w}{\sqrt{2 \pi} w y_{0}}=\frac{A}{y_{0}} .
$$

In the fitting area, the correlation coefficient is calculated with a sliding window to select the best splicing area where the correlation is the highest. We should point out that the backscatter lidar has constant resolution of $7.5 \mathrm{~m}$, whereas the resolution of the CCD imaging lidar varies with altitudes. So we need to compare the resolution of the two groups of signals and interpolate the lower one to another before 


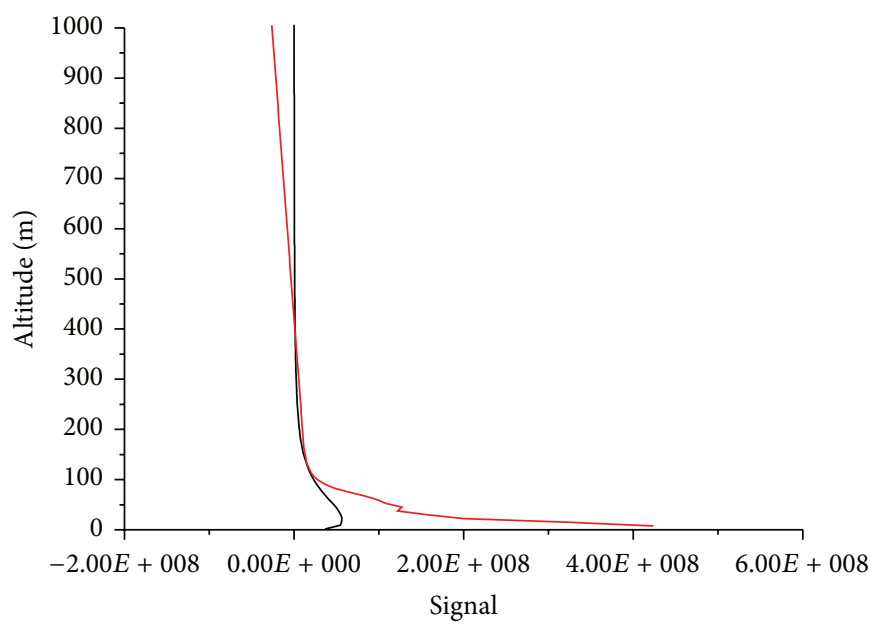

2014-3-22

— Backscatter lidar

_ CCD camera

(a)

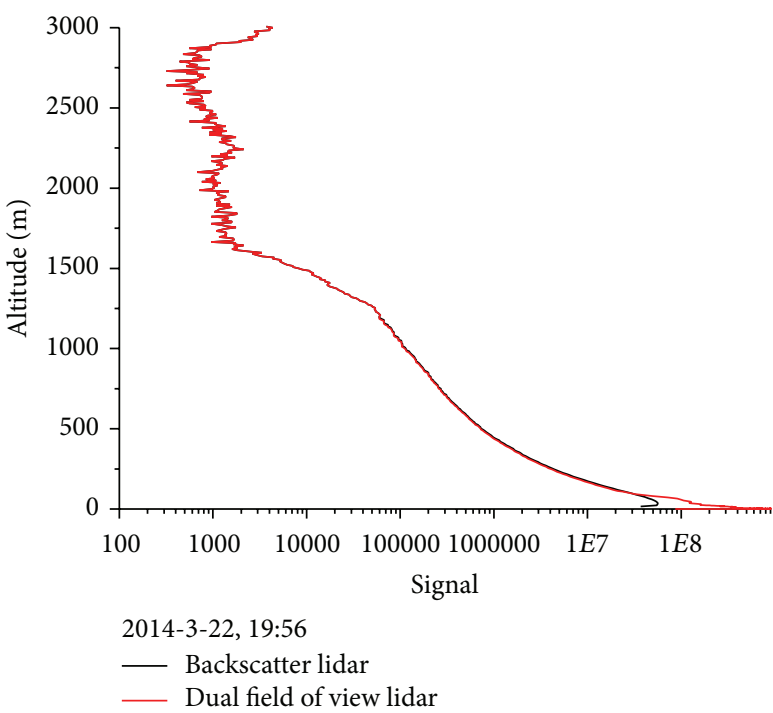

(c)

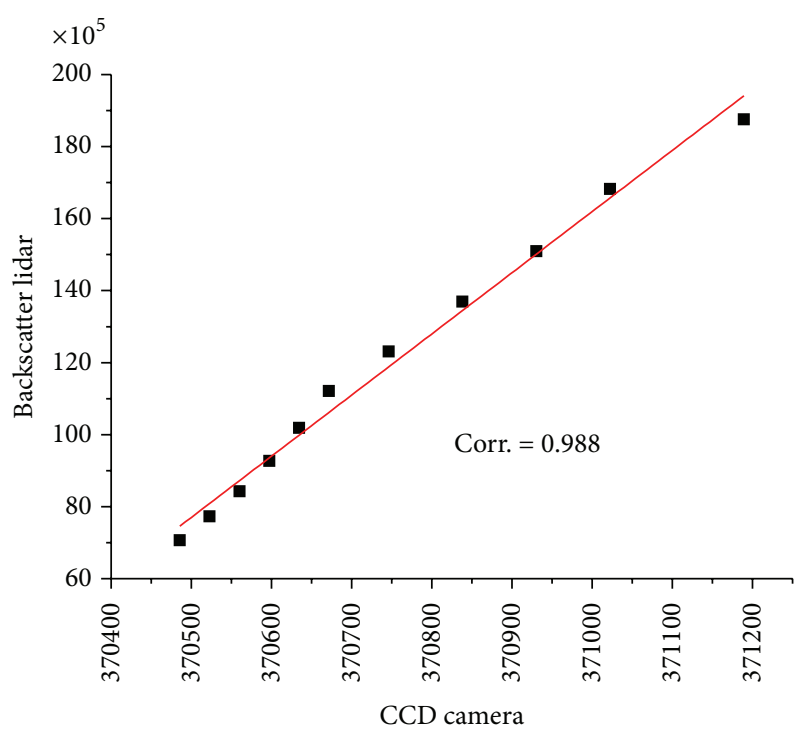

(b)

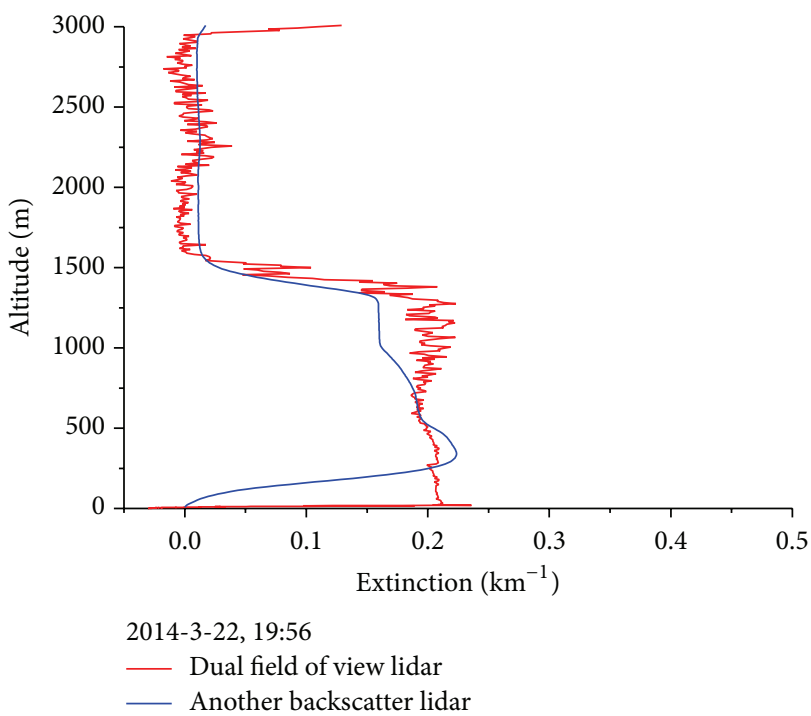

(d)

FIGURE 5: (a) The original signals received by the two receivers of the dual field of view lidar. (b) The correlation analysis in the range of 220-300 m. (c) Comparison of the gluing signal and original backscatter lidar signal received by the telescope. (d) Comparison of extinction coefficient profiles.

calculating the correlation coefficient of each window. Finally, the two groups of signals are fitted in the best splicing area and the fitting parameters are applied to the whole signal of the CCD camera to splice it with the photo counting signal received by the backscatter lidar. The spliced signal not only includes the near-ground data, but also takes the detection range into account, so the method can detect the distribution of atmospheric aerosols effectively.

The Fernald algorithm is usually used to invert the aerosol profiles [12]. The key point of the algorithm is to determine the reference point and its initial value. The conventional Fernald algorithm often selects the clear aerosol-free altitude of the atmosphere as the reference point and calculates its initial value with the standard atmospheric model.
But sometimes the clear altitude does not exist in the detection path, so here an improved Fernald algorithm is utilized to invert the aerosol extinction coefficient. Specifically the subslope method is used to determine the reference point and its initial value, and then the result is substituted into the Fernald formula to solve the aerosol profile [13].

\section{Experimental Results}

The observation experiment was carried out at night to avoid the impact of background. Figure 5(a) shows the original signals received by the two receivers of the dual field of view lidar. As shown in Figure 5(a), the signals of the two receivers have a good agreement in the range of $220-300 \mathrm{~m}$. 


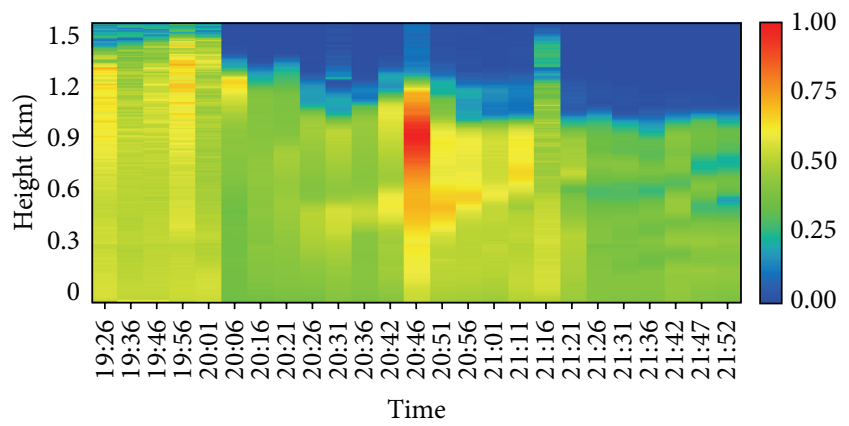

FIGURE 6: The distribution of aerosol extinction coefficient on March 22.

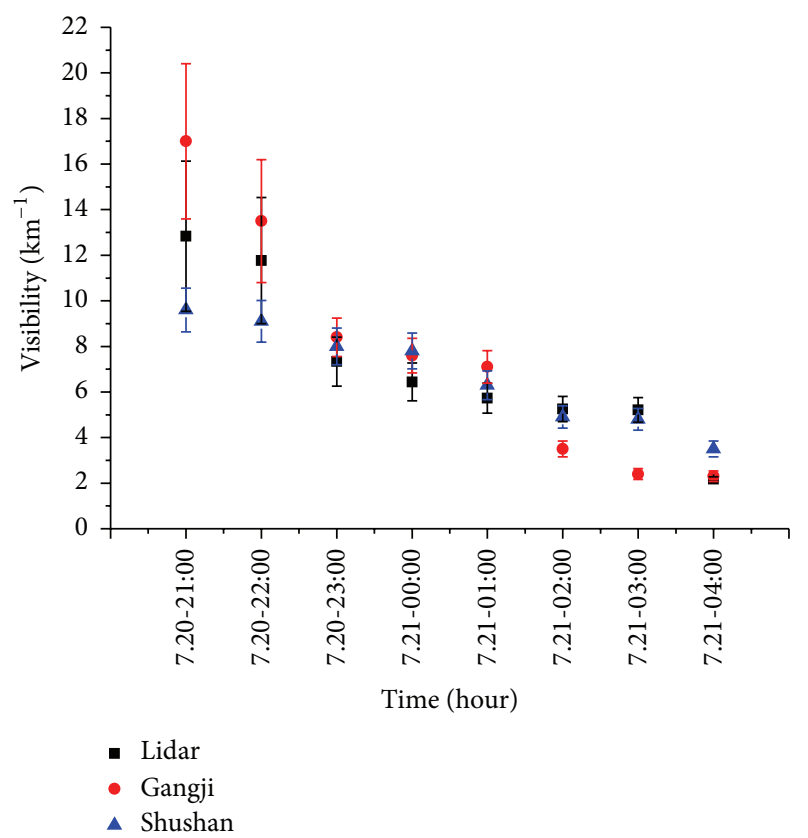

FIGURE 7: The comparison diagram of the visibility measured by the dual field of view lidar and forward scatter visibility meter.

The correlation analysis result shown in Figure 5(b) suggests that this area can be selected as the fitting area. Figure 5(c) shows the comparison of the gluing signal and the original backscatter lidar signal received by the telescope. Figure 5(d) shows the profiles of aerosol extinction coefficient retrieved by the dual field of view lidar system and another backscatter lidar which was placed near the dual field of view lidar. The final result shows that the splicing signal can inverse the aerosol distribution effectively in the near range.

Figure 6 presented the distribution of aerosol extinction coefficient from 19:30 to 22:00 on March 22. During the experiment the extinction coefficient decreased gradually in the period from 19:30 to 20:30 and increased but then decreased gradually in the range of $0.3-1.2 \mathrm{~km}$ later. In addition, the figure shows that the result inverted from the splicing signal presents a better distribution of aerosols in

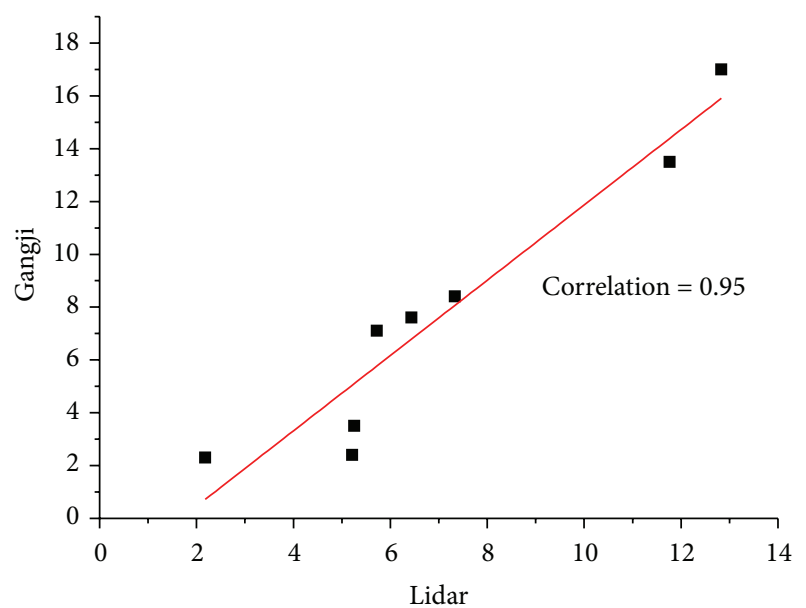

(a)

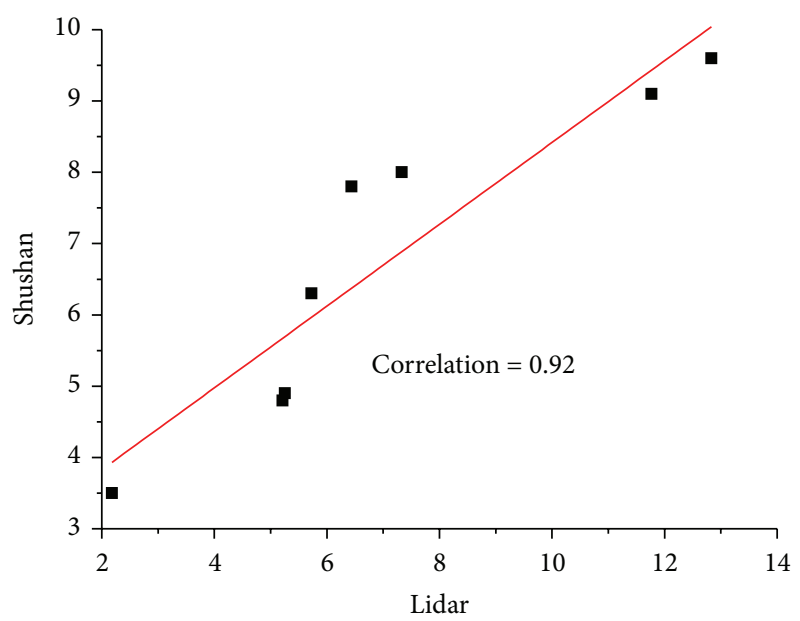

(b)

FIGURE 8: (a) The correlation analysis of the visibility measured by the dual field of view lidar and a forward scatter visibility meter located at Gangji station. (b) The correlation analysis of the visibility measured by the dual field of view lidar and a forward scatter visibility meter located at Shushan station.

the lower hundreds of meters, indicating the advantages of the dual field of view lidar system.

Furthermore the visibility measured by the dual field of view lidar was compared with the ones from a forward scatter visibility meter. In Figure 7, the square data represents the visibility [14] measured by the dual field of view lidar, and the circular and triangular data represent the visibility of Gangji and Shushan stations. The stations are all located within a radius of 6 kilometers from the site of the lidar. From the figure, we can see that the three curves are all tending to decline and the results have little difference during the experimental period. Figures 8 (a) and 8 (b) further, respectively, show that the correlativity of the visibility measured by the dual field of view lidar system and forward scatter visibility meter is above $90 \%$, so the dual field of view lidar can measure the visibility accurately and effectively. 


\section{Conclusion}

In conclusion, we introduce a new method for the aerosol detection with a dual field of view lidar system. A CCD camera is used to detect the lower atmosphere and a backscatter lidar is used to detect the higher atmosphere; finally the signals are spliced to invert the extinction coefficient profiles. CCD camera has the characteristics of high dynamic and high resolution near-ground, compensating the data of the blind and transition zone of the backscatter lidar. The signal spliced not only includes the near-ground data, but also takes the detection range into account, so the method can detect the distribution of atmospheric aerosols effectively. In addition, the improved Fernald algorithm is used to inverse the extinction coefficient profiles and the result is compared with the previous algorithm. The final result suggests that the improved algorithm is reasonable and effective. We have basically realized the no-blind aerosol detection with the dual field of view lidar system. Currently the dual field of view lidar technique is limited to use in night, so a further solution should be discussed to solve the background during the day.

\section{Conflict of Interests}

The authors declare that there is no conflict of interests regarding the publication of this paper.

\section{Acknowledgments}

This work was supported by the National Natural Science Foundation of China (no. 41305126), National Natural Science Foundation of China (no. 41205119), Key Project of Chinese National Programs for Fundamental Research and Development (973 Program) (Grant 2014CB447900), and the State Plan for High-Tech Research and Development (863 Program) (Grant 2014AA06A512).

\section{References}

[1] C. Weitkamp, Lidar: Range-Resolved Optical Remote Sensing of the Atmosphere, vol. 102 of Springer Series in Optical Sciences, Springer, New York, NY, USA, 2005.

[2] T. Halldorsson and J. Langerholc, "Geometrical form factors for the lidar function," Applied Optics, vol. 17, no. 2, pp. 240-244, 1978.

[3] B. M. Welsh and C. S. Gardner, "Bistatic imaging lidar technique for upper atmospheric studies," Applied Optics, vol. 28, no. 1, pp. 82-88, 1989.

[4] K. Meki, K. Y. Yamaguchi, X. Li, Y. Saito, T. D. Kawahara, and A. Nomura, "Range-resolved bistatic imaging lidar for the measurement of the lower atmosphere," Optics Letters, vol. 21, no. 17, pp. 1318-1320, 1996.

[5] J. E. Barnes, S. Bronner, R. Beck, and N. C. Parikh, "Boundary layer scattering measurements with a charge-coupled device camera lidar,' Applied Optics, vol. 42, no. 15, pp. 2647-2652, 2003.

[6] Z. Wang, Z. Tao, D. Liu, D. Wu, C. Xie, and Y. Wang, "New experimental method for lidar overlap factor using a CCD sidescatter technique," Optics Letters, vol. 40, no. 8, pp. 1749-1752, 2015.
[7] S.-H. Wang, M.-F. Hu, and Z.-P. Chen, "Noise analysis and the study of SNR model on the astronomical CCD camera," Semiconductor Optoelectronics, vol. 28, no. 5, pp. 731-734, 2007.

[8] E. Vermote, Second Simulation of the Satellite Signal in the Solar Spectrum, 6S User Guide Version 2, 1997.

[9] D. N. Whiteman, B. Demoz, P. Di Girolamo et al., "Raman lidar measurements during the international $\mathrm{H}_{2} \mathrm{O}$ project. Part I: instrumentation and analysis techniques," Journal of Atmospheric and Oceanic Technology, vol. 23, no. 2, pp. 157-169, 2006.

[10] H. Lifeng, G. Wei, L. Jun et al., "Signal splicing of dual-receiver Mie scattering lidar in atmospheric remote sensing," Journal of Remote Sensing, vol. 16, no. 4, pp. 712-719, 2012.

[11] J. E. Barnes, N. C. Parikh Sharma, and T. B. Kaplan, "Atmospheric aerosol profiling with a bistatic imaging lidar system," Applied Optics, vol. 46, no. 15, pp. 2922-2929, 2007.

[12] F. G. Fernald, "Analysis of atmospheric lidar observations: some comments," Applied Optics, vol. 23, no. 5, pp. 652-653, 1984.

[13] Q. Du, Y. Ji, and C. Xu, "Data processing method of aerosol horizontal distribution detected by MPL," Journal of Atmospheric and Environmental Optics, vol. 3, no. 1, pp. 23-27, 2008.

[14] X. Chengbo, H. Yong, L. Chao et al., "The vehicle-mounted Lidar used for atmospheric horizontal visibility measurement," High Power Laser and Particle Beams, vol. 17, no. 7, pp. 971-975, 2005. 

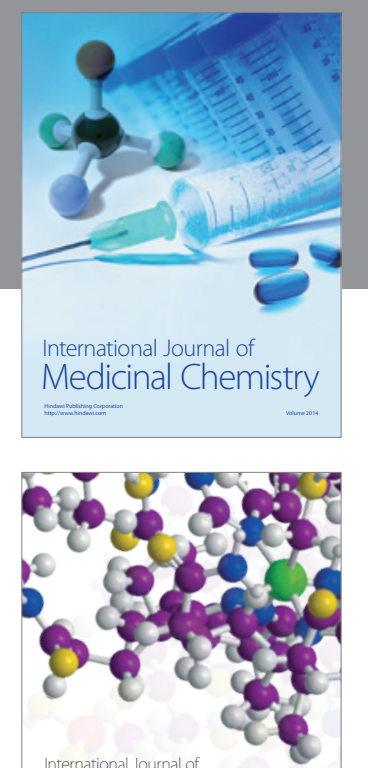

\section{Carbohydrate} Chemistry

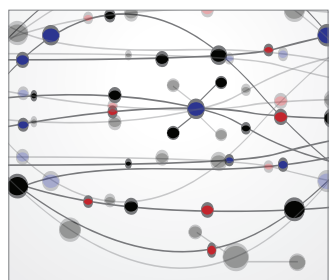

The Scientific World Journal
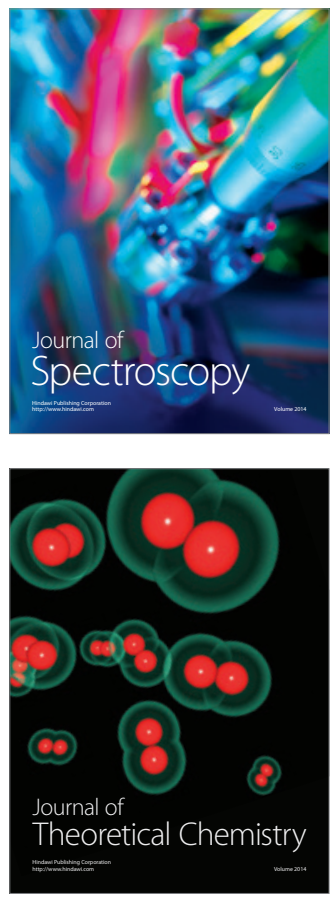
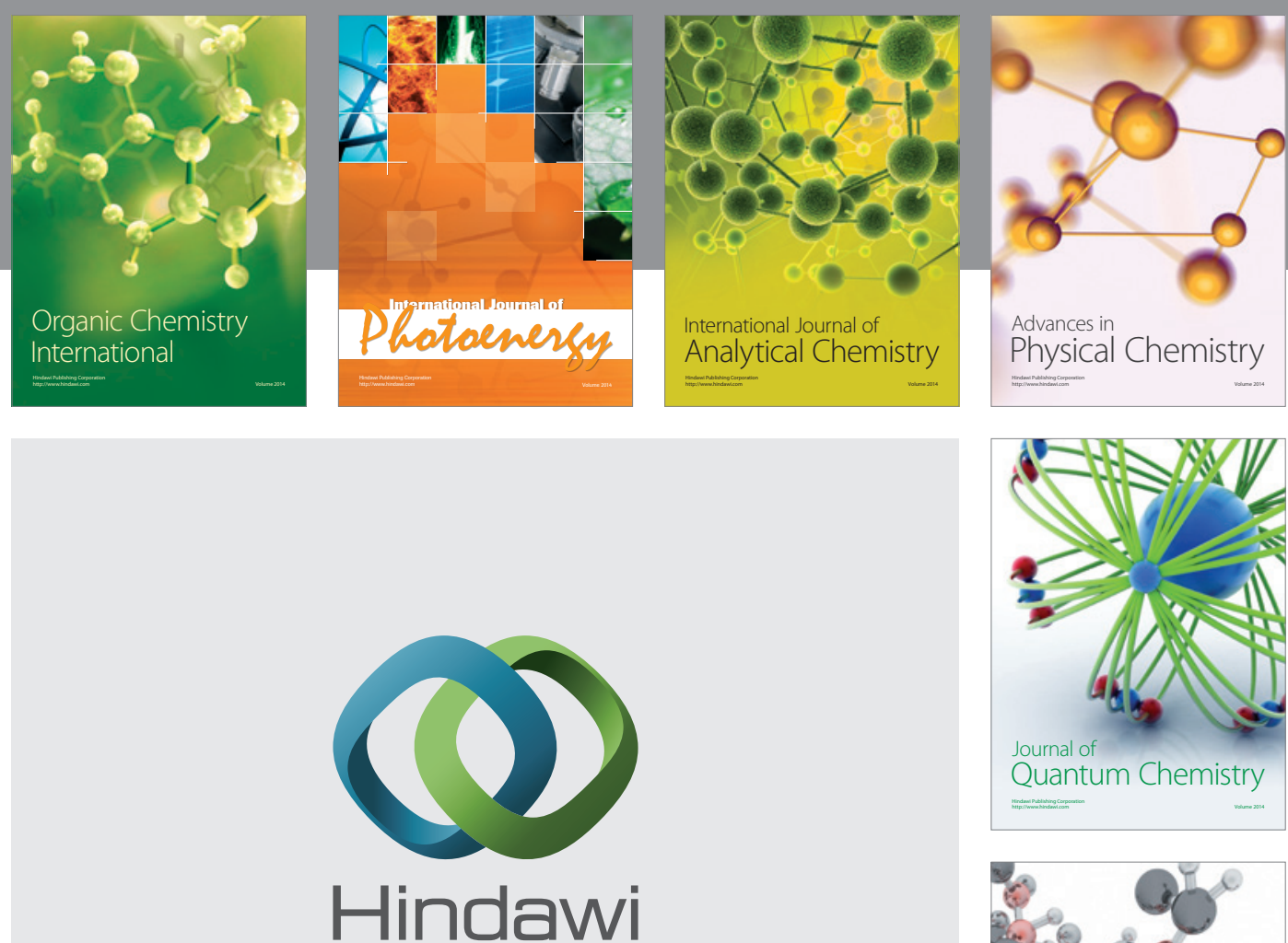

Submit your manuscripts at

http://www.hindawi.com

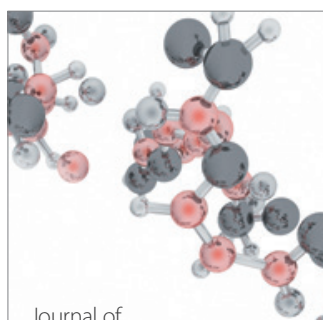

Analytical Methods

in Chemistry

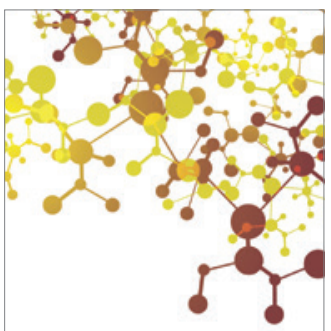

Journal of

Applied Chemistry

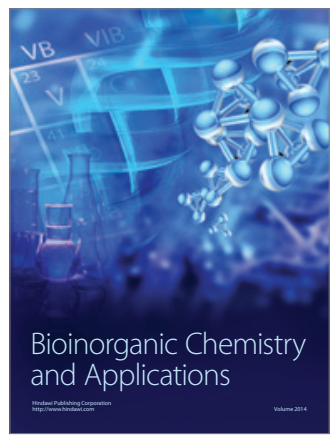

Inorganic Chemistry
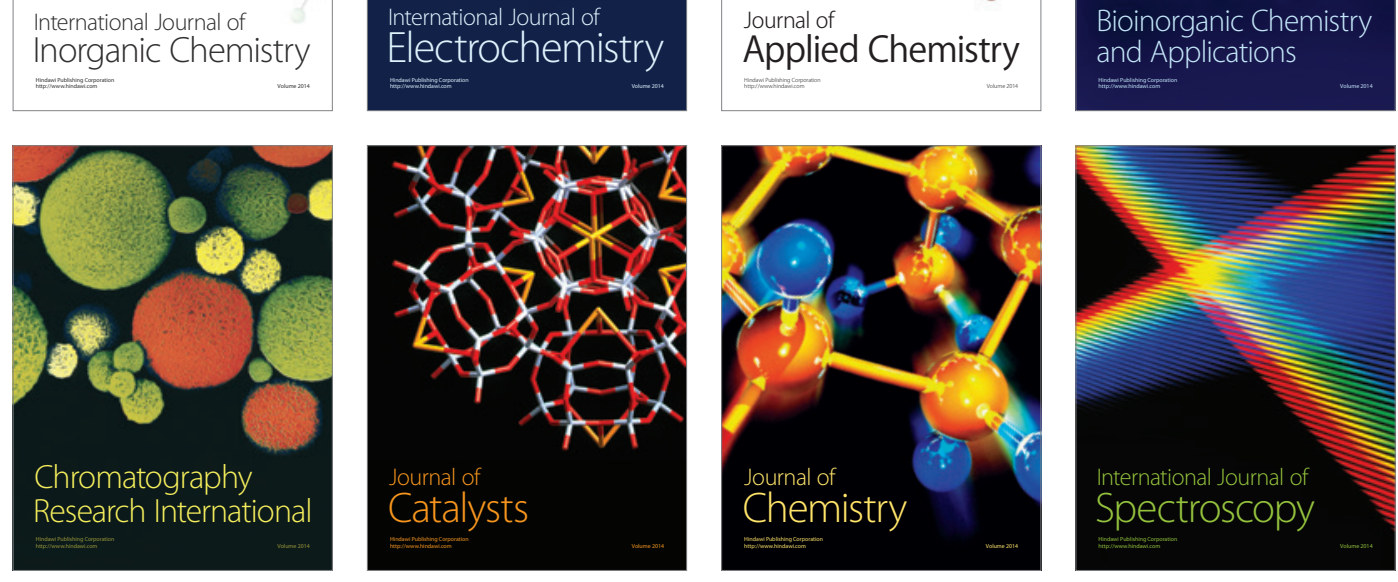